\title{
The correlations between $\mathrm{kHz}$ and $\mathrm{LF}$ QPOs in the NSXBs
}

\author{
Z. B. Li $^{1}$ and D. H. Wang ${ }^{2}$ \\ ${ }^{1}$ Xinjiang Astronomical Observatory, Chinese Academy of Sciences, \\ 150, Science 1-Street, Urumqi, Xinjiang, 830011, China \\ email: lizhibing@xao.ac.cn \\ ${ }^{2}$ National Astronomical Observatories, Chinese Academy of Sciences, \\ Bejing, 100012, China
}

\begin{abstract}
We study the correlations between low frequency (LF) and $\mathrm{kHz}$ quasi-periodic oscillations (QPOs) for 20 neutron star X-ray bianaries (NSXBs). We find that both upper and lower $\mathrm{kHz}$ QPOs correlate with the LF QPOs. However, the correlations show 5 parallel branches in the $\mathrm{kHz}$ QPO frequency vs. LF QPO frequency relation diagram. We notice that the source will jump from one parallel branch to another when different harmonic frequency of the LF QPO is used to plot the correlation between $\mathrm{kHz}$ and LF QPOs. We infer that the parallel branches maybe due to the multi-harmonic QPOs in the NSXBs.
\end{abstract}

Keywords. Accretion, stars: neutron, stars: oscillations, X-rays: binaries

\section{Introduction}

The kHz QPO is firstly found in Sco X-1 by RXTE (van der Klis et al. 1996). To date, about 35 NSXBs show kHz QPOs (Zhang et al. 2012). These kHz QPOs often show twin peaks, named as lower and upper $\mathrm{kHz}$ QPOs. The frequency separation between the twin $\mathrm{kHz}$ QPOs typically varies by tens of $\mathrm{Hz}$ as the lower/upper $\mathrm{kHz}$ QPO frequency changes by hundreds of $\mathrm{Hz}$ for a single source. The ratio between frequencies of the upper and lower $\mathrm{kHz}$ QPOs often close to the $3 / 2$ value, but the other rational ratios occur in some sources as well (Zhang et al. 2006).

Various models are proposed to explain the $\mathrm{kHz}$ QPOs. Such as the sonic point and spin resonance model (Miller et al. 1998), orbital resonance model (Abramowicz et al. 2003), relativistic precession model (Stella and Vietri 1999), MHD Alfven-wave oscillation model (Zhang 2004) and so on. Although each model achieves success to a certain extent, the origin of $\mathrm{kHz}$ QPOs is still highly debated. In this work, we focus mainly on the correlations between $\mathrm{kHz}$ and LF QPOs and we hope this will help to distinguish or improve the QPO models.

\section{Observations and Results}

There are $35 \mathrm{X}$-ray sources reported to exhibit kHz QPOs, and only 20 sources whose $\mathrm{kHz}$ and LF QPOs frequencies are listed in the published papers. The characteristic frequency of QPOs in the literatures are turned into QPO frequency and the correlations between the frequencies of $\mathrm{kHz}$ and LF QPOs for the 20 sources are plotted in Fig. 1. The figure shows 5 parallel branches. (Except for Sco X-1, whose LF and kHz QPO frequencies are $13.64 \mathrm{~Hz}$ and $1129.70 \mathrm{~Hz}$ (van der Klis et al. 1996), which make it locating at the left top of the 5 branches.) The lowest and the upper half part of the 2nd lowest branches stand for the correlation between the frequencies of the lower $\mathrm{kHz}$ and the LF QPOs, while the remaining branches represent the correlation between the frequencies of the upper $\mathrm{kHz}$ and $\mathrm{LF}$ QPOs. 


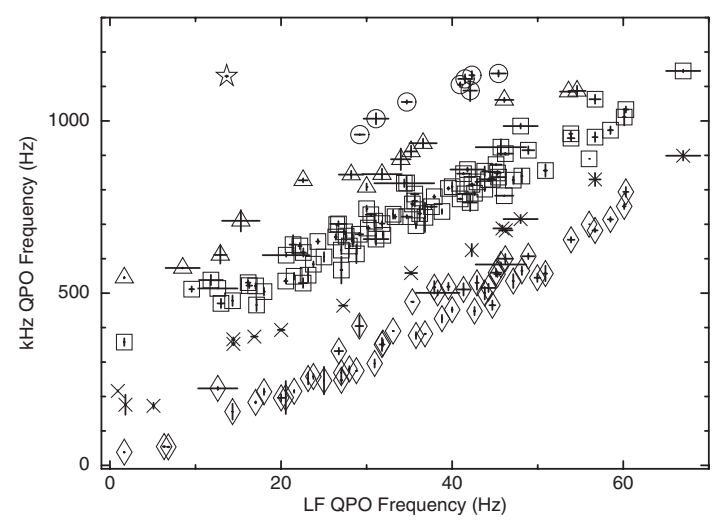

Figure 1. The correlations between the frequencies of $\mathrm{kHz}$ and LF QPOs. The lowest and the upper half part of the 2 nd lowest branch stand for the correlation between the lower $\mathrm{kHz}$ and LF QPOs, while the remain represent the correlation between the upper $\mathrm{kHz}$ and LF QPOs.

\section{Discussion}

We have studied the correlations between the frequencies of $\mathrm{kHz}$ and $\mathrm{LF}$ QPOs of $20 \mathrm{NSXBs}$ and found the lower $\mathrm{kHz}$ QPOs correlate with the LF QPOs. The result is consistent with that of Belloni et al. (2002). Moreover, the upper $\mathrm{kHz}$ QPOs also correlate with the LF QPOs in the manner of several parallel branches. A single source can simultaneously appear at more than one branch, suggesting that the different branches are not due to the source types.

The NSXBs' LF QPOs usually have a few harmonics and if the harmonic frequency is used to measure the correlation it will jump from one branch to another. For example, in one case of Cyg X-2, the LF QPO at $28.4 \mathrm{~Hz}$ has a sub-harmonic at $14.19 \mathrm{~Hz}$ and a first harmonic at $55.04 \mathrm{~Hz}$, and its $\mathrm{kHz}$ QPO is at $652.30 \mathrm{~Hz}$ (Kuznetsov 2002). When the $14.19 \mathrm{~Hz}$ is used to measure the correlation, it locate at the 2nd topmost branch. However, the $28.4 \mathrm{~Hz}$ (or $55.04 \mathrm{~Hz}$ ) QPO makes it locate at the middle (or lowest) branch. Moreover, for Sco X-1, if its LF QPO has a third harmonic at about $41 \mathrm{~Hz}$ and use the third harmonic to measure the correlation, it will locate at the uppermost branch. As a result, we infer that it is the multi-harmonics of the LF QPO causes the parallel branches in the correlations between the frequencies of the $\mathrm{kHz}$ and LF QPOs.

\section{Acknowledgments}

This work is subsidized by the Program of the Light in Chinese Western Region (LCWR) (Grant No. XBBS201121) provided by Chinese Academy of Sciences (CAS), the Natural Science Foundation of China for Young Scientists (Grant No. 11203064) and the Natural Science Foundation of China via NSFC 1143013, 11173024 and 11173034.

\section{References}

Abramowicz, M. A., Karas, V., Kluzniak, W., Lee, W. H., \& Rebusco, P. 2003, PASJ, 55, 467

Belloni, T., Psaltis, D., \& van der Klis, M. 2002, ApJ, 572, 392

Kuznetsov S. I. 2002, Astron. Lett., 28, 73

Miller, M. C., Lamb, F. K., \& Psaltis, D. 1998, ApJ, 508, 791

van der Klis, M., Swank, J. H., Zhang, W., Jahoda, K., Morgan, E. H. et al. 1996, ApJ, 469, L1 Stella, L. \& Vietri, M. 1999, Phys. Rev. Lett., 82, 17

Zhang, C. M., Pan, Y. Y., Wang, J., Taani, A., \& Zhao, Y. H. 2012, Int. J. Mod. Phys. Conf. Ser., 12,414

Zhang, C. M. 2004, A\&A, 423, 401

Zhang, C. M., Yin, H. X., Zhao, Y. H., Song, L. M., \& Zhang, F. 2006, MNRAS, 366, 1373 